\title{
Chaos in driven Alfvén systems: unstable periodic orbits and chaotic saddles
}

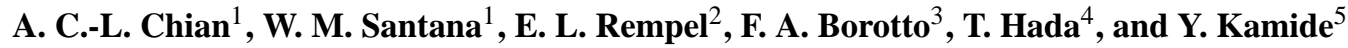 \\ ${ }^{1}$ National Institute for Space Research (INPE) and World Institute for Space Environment Research (WISER), P.O. Box 515, \\ São José dos Campos - SP, CEP 12227-010, Brazil \\ ${ }^{2}$ Institute of Aeronautical Technology (ITA), São José dos Campos - SP, CEP 12228-900, Brazil \\ ${ }^{3}$ Universidad de Concepción, Departamento de Física, Concepción, Chile \\ ${ }^{4}$ Kyushu University, Department of Earth Sciences and Technology, Fukuoka 8168580, Japan \\ ${ }^{5}$ Solar-Terrestrial Environment Laboratory, Nagoya University, Toyokawa 4428507, Japan
}

Received: 7 April 2006 - Revised: 8 January 2007 - Accepted: 8 January 2007 - Published: 24 January 2007

\begin{abstract}
The chaotic dynamics of Alfvén waves in space plasmas governed by the derivative nonlinear Schrödinger equation, in the low-dimensional limit described by stationary spatial solutions, is studied. A bifurcation diagram is constructed, by varying the driver amplitude, to identify a number of nonlinear dynamical processes including saddlenode bifurcation, boundary crisis, and interior crisis. The roles played by unstable periodic orbits and chaotic saddles in these transitions are analyzed, and the conversion from a chaotic saddle to a chaotic attractor in these dynamical processes is demonstrated. In particular, the phenomenon of gap-filling in the chaotic transition from weak chaos to strong chaos via an interior crisis is investigated. A coupling unstable periodic orbit created by an explosion, within the gaps of the chaotic saddles embedded in a chaotic attractor following an interior crisis, is found numerically. The gapfilling unstable periodic orbits are responsible for coupling the banded chaotic saddle (BCS) to the surrounding chaotic saddle (SCS), leading to crisis-induced intermittency. The physical relevance of chaos for Alfvén intermittent turbulence observed in the solar wind is discussed.
\end{abstract}

\section{Introduction}

Unstable structures such as unstable periodic orbits and chaotic saddles play a fundamental role in nonlinear dynamics of the earth-ocean-space environment (Samelson, 2001a; Chian et al., 2003; Selten and Branstator, 2004), economics (Ishiyama and Saiki, 2005; Chian et al., 2006), fluid mechanics (Kawahara and Kida, 2001; Faisst and Eckhard, 2003),

Correspondence to: A. C.-L. Chian

(achian@dge.inpe.br) lasers (Green and Krauskopf, 2002), and neuroscience (Harrison et al., 2004).

Unstable periodic orbits are the skeleton of a chaotic attractor since its chaotic trajectory is the closure of an infinite set of unstable periodic orbits (Ott, 1993; Auerbach et al., 1987; Cvitanović, 1988). In contrast to a periodic attractor thereby all trajectories initiated from any point in the state space within its basin of attraction are attracted to a stable periodic orbit, in a chaotic attractor all periodic orbits are unstable. Chaotic sets are not necessarily attracting sets. A set of unstable periodic orbits can be chaotic and nonattracting so that the orbits in the neighborhood of this set are eventually repelled from it; nonetheless, this set can contain a chaotic orbit with at least one positive Lyapunov exponent; if the chaotic orbit has also one negative Lyapunov exponent the nonattracting set is known as a chaotic saddle (Grebogi et al., 1983; Nusse and Yorke, 1989; Szabó and Tél, 1994a,b).

Recently, there has been a remarkable advance in the study of unstable periodic orbits and chaotic saddles in fluid turbulence. Christiansen et al. (1997) and Zoldi and Greenside (1998) showed that the periodic orbit theory of dynamical systems formulated by Auerbach et al. (1987) and Cvitanović (1988) can determine the global averages of a chaotic attractor or a chaotic saddle in the Kuramoto-Sivashinsky equation, based on a finite ensemble of fundamental unstable periodic orbits. Kawahara and Kida (2001) numerically found an unstable periodic orbit in a three-dimensional plane Couette turbulence described by the incompressible Navier-Stokes equation. (Chian et al., 2002) and Rempel et al. (2004a) showed that unstable periodic orbits and chaotic saddles can characterize an interior crisis and the intermittency induced by an interior crisis in the Kuramoto-Sivashinsky equation. Faisst and Eckhard (2003) identified a family of unstable traveling waves originating from saddle-node bifurcations in a

Published by Copernicus GmbH on behalf of the European Geosciences Union and the American Geophysical Union. 
numerical experiment of flow through a pipe, and showed that these unstable structures provide a skeleton for the formation of a chaotic saddle responsible for the transition to turbulence. Kato and Yamada (2003) detected an unstable periodic orbit in the Gledzer-Ohkitani-Yamada shell model turbulence, and showed that an intermittent turbulence is described by this unstable periodic orbit. Kawahara (2005) applied the method of controlling chaos to stabilize an unstable periodic orbit in a plane Couette turbulent flow and succeeded in driving the system to a regime of laminar flow. Rempel and Chian (2005) showed that unstable periodic orbits and chaotic saddles are the origin of the intermittency induced by an attractor merging crisis in the Kuramoto-Sivashinsky equation. Van Veen et al. (2006) extracted unstable periodic orbits from a forced box turbulence with high symmetry and compared the statistical properties of these periodic flows with those of turbulent flow.

The aim of this paper is to investigate the roles of unstable periodic orbits and chaotic saddles in nonlinear processes in space plasmas. Based on a low-dimensional model of driven Alfvén systems originally formulated by Hada et al. (1990), Chian et al. (1998) showed that a nonlinear Alfvén wave in the solar wind can evolve from order to chaos via a saddlenode bifurcation and evolve from weak chaos to strong chaos via an interior crisis. Borotto et al. (2004) identified numerically a period-9 unstable periodic orbit responsible for the Alfvén chaos studied by Chian et al. (1998), leading to the onset of a boundary crisis and an interior crisis. Following the works of Hada et al. (1990), Chian et al. (1998) and Borotto et al. (2004), we show in this paper that local and global bifurcations such as saddle-node bifurcation and boundary/interior crises involve the conversion of a chaotic saddle into a chaotic attractor. This conversion may result from gap-filling due to the creation of new unstable periodic orbits in the gap regions of the chaotic saddles. An example of gap-filling unstable periodic orbit in an interior crisis is numerically determined.

Crisis results from a global bifurcation whereby a chaotic attractor suddenly widens or disappears (Grebogi et al., 1983). The former is known as an interior crisis and the latter a boundary crisis. Both types of crisis involve the collision of a chaotic attractor with an unstable periodic orbit or its stable manifold. We will demonstrate in this paper that at the onset of both boundary crisis and interior crisis, a chaotic attractor collides with a chaotic saddle.

Section 2 of this paper formulates the derivative nonlinear Schrödinger equation that models the propagation of a nonlinear Alfvén wave. Section 3 analyzes chaos in Alfvén systems based on bifurcation diagrams, unstable periodic orbits, chaotic attractors and chaotic saddles. Section 4 discusses the saddle-node bifurcation. Section 5 treats the boundary crisis. Section 6 studies the interior crisis. Discussion and conclusion are given in Sect. 7 .

\section{Derivative nonlinear Schrödinger equation}

Nonlinear spatiotemporal evolution of Alfvén waves can be modeled by the derivative nonlinear Schrödinger equation (DNLS) (Hada et al., 1990; Chian et al., 1998; Borotto et al., 2004):

$\partial_{t} b+\alpha \partial_{x}\left(|b|^{2} b\right)-i(\mu+i \eta) \partial_{x}^{2} b=S(b, x, t)$,

where the wave is propagating along an ambient magnetic field $B_{0}$ in the x-direction, $b=b_{y}+i b_{z}$ is the complex transverse wave magnetic field normalized to the constant ambient magnetic field, $\mu$ is the dispersive parameter, $\eta$ is a characteristic scale length, time $t$ is normalized to the inverse of the ion cyclotron frequency $\omega_{c i}=e B_{0} / m_{i}$, space $x$ is normalized to $c_{A} / \omega_{c i}, c_{A}=B_{0} /\left(\mu_{0} \rho_{0}\right)^{1 / 2}$ is the Alfvén velocity, $c_{S}=\left(\gamma P_{0} / \rho_{0}\right)^{1 / 2}$ is the acoustic velocity, $\alpha=1 /[4(1-\beta)]$, and $\beta=c_{S}^{2} / c_{A}^{2}$. The external forcing $S(b, x, t)=A \exp (i k \phi)$ is a monochromatic left-hand circularly polarized wave with a wave phase $\phi=x-V t$, where $V$ is a constant wave velocity, $A$ is the driver amplitude, and $k$ is the driver wave number.

Equation (1) has been extensively used to study the nonlinear evolution of Alfvén waves and MHD phenomena. Mjølhus (1976) used DNLS to examine the modulational instability of circularly polarized MHD waves of finite amplitude propagating parallel to the ambient magnetic field. Spangler and Sheerin (1982) derived DNLS from two-fluid equations using the reductive perturbation method and analyzed the properties of an envelope Alfvén soliton based on the pseudo-potential formalism. Machida et al. (1987) compared the temporal behavior of the electromagnetic hybrid simulation with the numerical solution of DNLS, which shows that a left-hand circularly polarized Alfvén mode evolves into a shocklike structure due to the modulational instability; for the right-hand mode, the formation of shock does not take place. Kennel et al. (1988) derived DNLS by means of Lagrangian variables and obtained solitary and periodic solutions of elliptically polarized quasiparallel Alfvén waves. Hada et al. (1989) used a pseudo-potential method to classify the stationary solutions of DNLS, which consist of a rich family of nonlinear Alfvén waves and solitons with parallel and oblique propagations. Dawson and Fontan (1990) compared two statistical models of Alfvén solitons described by DNLS and applied the resulting power spectra to solar wind observations. Buti (1991) showed that nonlinear Alfvén waves in inhomogeneous plasmas are governed by a modified DNLS; the inhomogeneity leads to acceleration/deceleration of Alfvén solitons. Spangler and Plapp (1992) performed numerical investigations of DNLS, which indicate that for circularly polarized wave packets close to parallel propagation there is little change in its wave form; however, for oblique propagation the wave steepens and undergoes polarization changes, and can generate highfrequency wavelets similar to the observed large-amplitude MHD waves upstream of the Earth's bow shock. Verheest and Buti (1992) used the reductive perturbation analysis to 
derive DNLS for parallel Alfvén waves in warm, streaming, multispecies plasmas. Khabibrakhmanov et al. (1993) developed a model of collisionless parallel shock based on a modified DNLS by including the anisotropy of the plasma distribution function and higher-order dispersion; the number of adiabatically reflected ions define the threshold conditions of the fire-hose and mirror-type instabilities in the upstream and downstream regions of the shock. Medvedev and Diamond (1996) modeled the kinetic resonant particle effects of nonlinear Alfvén waves by incorporating an additional term representing dissipation akin to parallel heat conduction in a modified DNLS, which removes the singularity usually encountered in the nonlinear terms of DNLS and takes into account nonlinear coupling of an Alfvénic mode to a kinetic ion-acoustic mode; damping of nonlinear Alfvén waves appears via a strong Landau damping of the ion-acoustic waves. Baumgartel (1999) applied the magnetically rarefactive (dark) MHD soliton solution of DNLS to explain magnetic holes observed in solar wind, planetary magnetosheath and cometary environment. Laveder et al. (2001) studied the transverse collapse of dispersive Alfvén wave trains by numerically solving a three-dimensional DNLS, which results in the formation of strong magnetic filaments along the ambient magnetic field. Ruderman (2002) showed that the oblique propagation of large-amplitude MHD solitons in a high-beta Hall plasma is described by DNLS. Krishan and Nocera (2003) studied the relaxed states of Alfvénic turbulence based on the spatiotemporal solutions of DNLS; they investigated the inverse energy cascade during four-wave interactions and concluded that Alfvénic turbulence relaxes to a state with soliton type structures which can become the constant magnetic field force-free state. Passot and Sulem (2003) obtained a criterion for filamentation instability of circularly polarized Alfvén wave train described by a generalized kinetic DNLS which retains Landau damping, derived from the Vlasov-Maxwell equations via long-wave reductive perturbative expansion. Verheest et al. (2004) conducted a comparative study of weakly nonlinear envelope soliton solutions of DNLS and large-amplitude stationary whistler oscillitons, by considering the role of charge neutrality. Chen and Lam (2004) and Lashkin (2005) applied the inverse scattering transform to DNLS to study the generation of Alfvén solitons.

In this paper, we adopt the low-dimensional model of nonlinear Alfvén waves (Hada et al., 1990; Chian et al., 1998; Borotto et al., 2004) to investigate the stationary spatial wave solutions of Eq. (1) with $b=b(\phi)$, whose first integral reduces to a set of three coupled ordinary differential equations describing the transverse wave magnetic fields and the wave phase of nonlinear Alfvén waves

$\dot{b}_{y}-v \dot{b}_{z}=\frac{\partial H}{\partial b_{z}}+a \cos \theta$,

$\dot{b}_{z}+v \dot{b}_{y}=-\frac{\partial H}{\partial b_{y}}+a \sin \theta$

$$
\dot{\theta}=\Omega,
$$

where $H=\left(\mathbf{b}^{2}-1\right)^{2} / 4-(\lambda / 2)\left(\mathbf{b}-\mathbf{e}_{y}\right)^{2}, b \rightarrow b / b_{0}$ (where $b_{0}$ is an integration constant), $\mathbf{b}=\left(b_{y}, b_{z}\right)$, the normalized driver amplitude parameter $a=A / \alpha b_{0}^{2} k$, the normalized damping parameter $\nu=\eta / \mu$, the overdot denotes derivative with respect to the wave phase $\tau=\alpha b_{0}^{2} \phi / \mu, \theta=\Omega \phi, \Omega=\mu k / \alpha b_{0}^{2}$, and $\lambda=-1+V / \alpha b_{0}^{2}$.

Equation (1) allows certain arbitrariness for choosing the signs of its various terms (Ghosh and Papadopoulos, 1987). The sign of the cubic nonlinear term depends on the plasma $\beta$. Here we assume $\beta<1$, hence $\alpha$ is positive. The sign of $\Omega$ depends on the polarization of the driver. We assume negative $\Omega$ for a left-hand circularly polarized driver. In addition, if the kinetic effects due to wave-particle interactions such as cyclotron/Landau damping or growth are included, a damping/growth operator appears in Eq. (1), which introduces a further flexibility with the sign. The inclusion of kinetic effects (e.g., nonlinear Landau damping), influences the sign of $\alpha$. It is no longer negative for $\beta>1$ unless the electron to ion temperature ratio is extremely large. The $\eta$ term in the partial differential equation Eq. (1) has to be treated with caution. An initial value problem based on Eq. (1), defined with exactly the same initial conditions but with different sign of $\eta$, will yield completely different time evolutions. By convention, the sign in front of $\eta$ in Eq. (1) should be negative if it is treated as a dissipation term. On the other hand, once the partial differential equation Eq. (1) is reduced to the set of ordinary differential equations Eqs. (2-4) by assuming the travelling wave solution $(\phi=x-V t)$, the phase variable $\phi$ then includes both space and time, hence the physical meaning above is lost. For example, the two opposite signs of $\eta$ now merely correspond to integrating from one end to the other in $\phi$, or the other way around. In fact, by re-defining $b=b_{y}-i b_{z}$ and $\phi=V t-x$, we obtain Eqs. (2-4) with the sign of $\eta$ reversed. Thus, the solution of $b$ as a function of $\phi$ remains essentially unchanged by flipping the sign of $\eta$. We can regard Eqs. (2-4) as a nonlinear model of driven-damped oscillator containing two control parameters $a$ and $v$. The sign of the control parameter $v$ depends on $\eta$ and the sign of the dispersion $\mu$. Here we assume $v$ is positive.

\section{Alfvén chaos}

In this paper, we analyze the roles played by unstable periodic orbits and chaotic saddles in driven Alfvén systems, based on the low-dimensional model of Alfvén chaos described by Eqs. (2-4).

A limit point diagram, which provides an overview of the system dynamics and its sensitive dependence on small variations in a system parameter, can be constructed from the numerical solutions of Eqs. (2-4) by varying the driver amplitude parameter $a$ while keeping other system parameters 
(a)

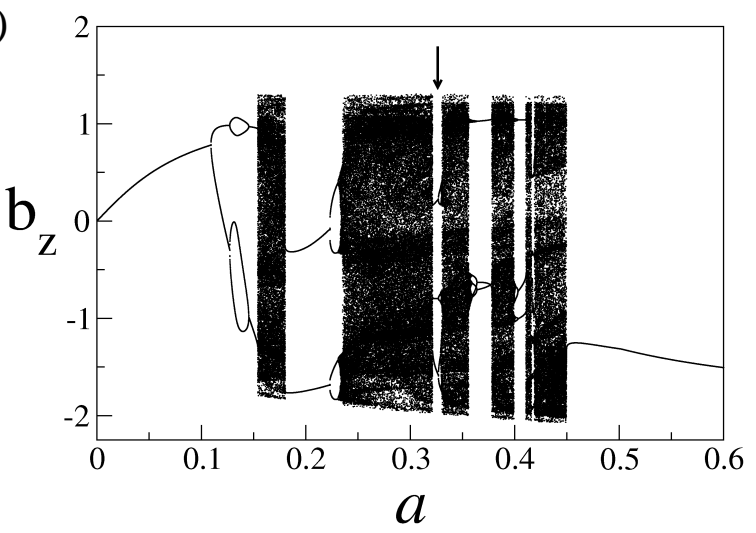

(b)

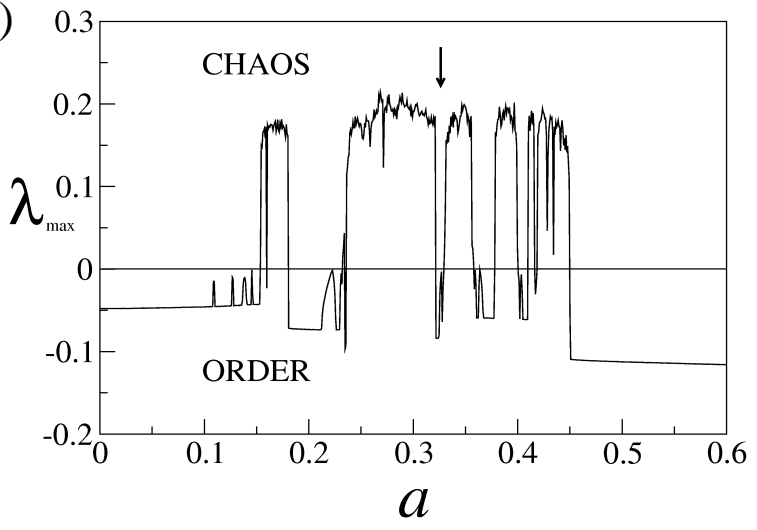

Fig. 1. Limit point diagram and maximum Lyapunov exponent: global view. (a) Limit point diagram, $b_{z}$ as a function of the driver amplitude $a$; (b) maximum Lyapunov exponent $\lambda_{\max }$ as a function of $a$. The arrow indicates a period-3 periodic window. $\nu=0.02$, $\Omega=-1, \lambda=1 / 4, \mu=1 / 2$.

fixed $(\nu=0.02, \Omega=-1, \lambda=1 / 4, \quad \mu=1 / 2)$. We define a Poincaré plane as

$$
P:\left[b_{y}(\tau), b_{z}(\tau)\right] \rightarrow\left[b_{y}(\tau+T), b_{z}(\tau+T)\right],
$$

where $T=2 \pi / \Omega$ is the driver period. Figure 1a displays a global view of the limit point diagram of nonlinear Alfvén waves. For a given $a$, Fig. 1a plots the asymptotic values of the Poincaré points of $b_{z}$, where the initial transient is omitted. Figure $1 \mathrm{~b}$ shows the maximum Lyapunov exponent as a function of $a$, for the limit point diagram of Fig. 1a, calculated by the Wolf algorithm (Wolf et al., 1985). Note that there are two algorithms in the paper by Wolf et al. (1985). The one based on the time series analysis has been improved by Kantz (1994), and another based on the integration of the Jacobian matrix of the flow which is a much more precise way to compute the Lyapunov spectrum. Since in the present paper we know the system equations, we adopt the second algorithm.

An enlargement of a small region of the limit point diagram indicated by the arrow in Fig. 1a is given in Fig. 2a, which displays both attractor (black) and chaotic saddle (blue) for a period-3 periodic window. Our choice of this periodic window is motivated by the pioneer paper by $\mathrm{Li}$ and York (1975) which states that period-3 implies chaos. Two attractors $A_{1}$ and $A_{2}$ are found within this periodic window. For attractor $A_{1}$, this periodic window begins with a saddlenode bifurcation (SNB) at $a_{\mathrm{SNB}}=0.321383$, where a pair of period-3 stable and unstable periodic orbits are created. The period-3 stable periodic orbit undergoes a cascade of perioddoubling bifurcations as $a$ increases and turns eventually into a banded chaotic attractor with three bands. This periodic window ends with an interior crisis (IC) at $a_{\mathrm{IC}}=0.330248$. To plot the chaotic saddle, for each value of $a$, we plot a straddle trajectory close to the chaotic saddle using the PIM triple algorithm with a precision of $10^{-6}$ (Nusse and York, 1989; Rempel and Chian, 2004; Rempel et al., 2004a). The blue region inside the periodic window in Fig. 2a, denotes the surrounding chaotic saddle (SCS) which acts as the transient preceding the convergence of the solutions to a periodic or a chaotic attractor; the surrounding chaotic saddle extends to the chaotic regions outside the periodic window, to the left of SNB and to the right of IC, where it becomes a subset of the chaotic attractor. After the interior crisis (IC), the banded chaotic attractor is converted into a banded chaotic saddle (red), as shown in Fig. 2b. In Fig. 2c, we plot the variation of the maximum Lyapunov exponent of the attractor $A_{1}$ as a function of $a$. Note that the value of the maximum Lyapunov exponent jumps suddenly at SNB and IC, implying an abrupt increase in the degree of chaoticity in the nonlinear Alfvén system.

Figure 2a indicates that the attractor $A_{2}$ appears in a narrow region within the p-3 periodic window. Figure 3 shows an enlargement of the region of $A_{2}$. The attractor $A_{2}$ begins at $a_{\mathrm{SNB}}=0.32935$, where a pair of stable and unstable periodic orbits of period- 9 are created due to a saddlenode bifurcation (SNB). The p-9 stable periodic orbit undergoes a cascade of period-doubling bifurcations, leading to the formation of a banded chaotic attractor with nine bands. This chaotic attractor is destroyed by a boundary crisis (BC) at $a_{\mathrm{BC}}=0.329437$. After BC, the banded chaotic attractor is converted into a banded chaotic saddle (red), as seen in Fig. 3a. The variation of the maximum Lyapunov exponent of the attractor $A_{2}$ as a function of $a$ is plotted in Fig. $3 \mathrm{~b}$.

Unstable periodic orbits in a chaotic system have specific functions in the system dynamics. For example, Fig. 4 shows an example of the trajectory (solid line) and the Poincare points (cross) of the period-9 unstable periodic orbit (M) created at $a_{\mathrm{SNB}}=0.32935$, which is responsible for mediating the onset of a boundary crisis (BC) in Fig. 3a as well as the onset of an interior crisis (IC) in Fig. 2a. The unstable periodic orbit is found by the Newton method (Curry, 1979).

Following Green and Krauskopf (2002), the dynamical behavior of Alfvén boundary and interior crises under investigation can be clarified by a detailed bifurcation diagram shown in Fig. 5. An attractor $A_{1}$ exists in the entire range of control parameter $a$ in Fig. 5a, which can be chaotic as well 
(a)

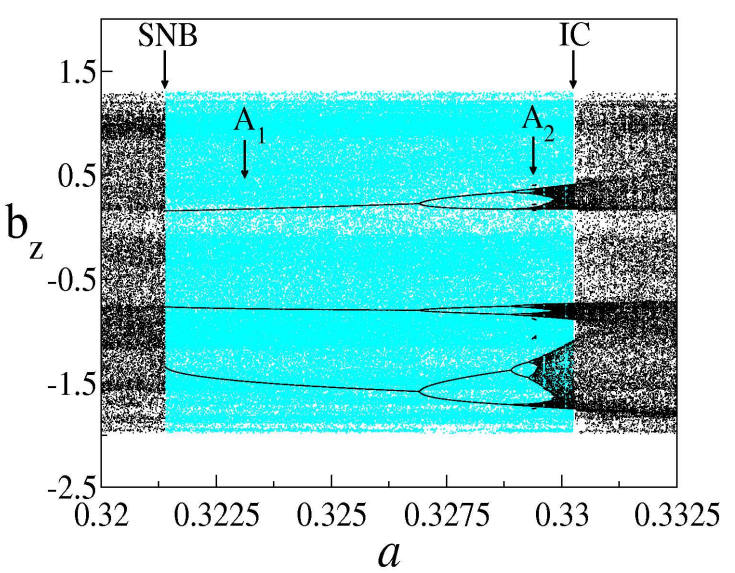

(b)

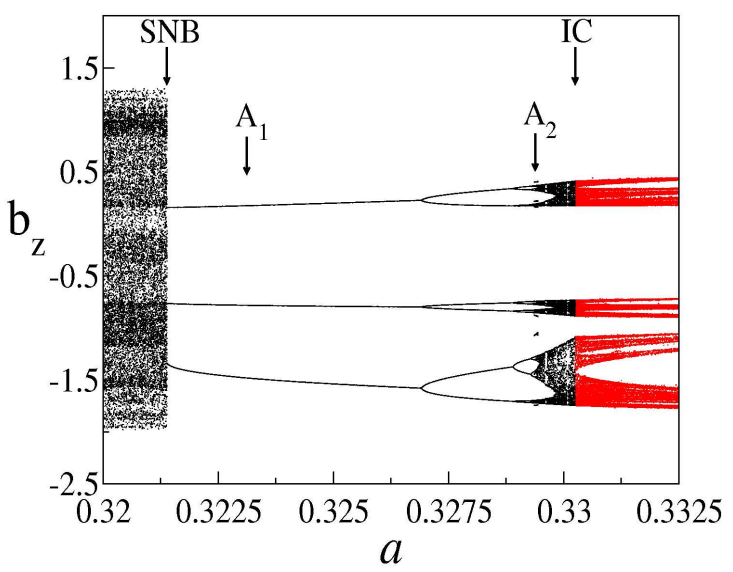

(c)

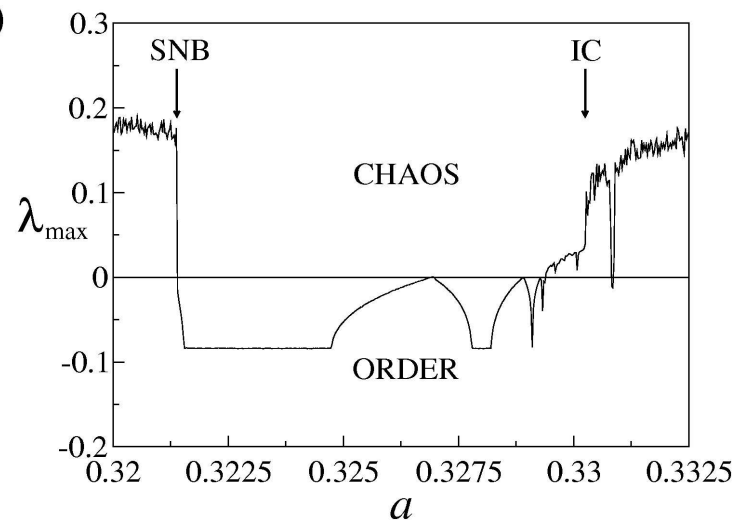

Fig. 2. Limit point diagram and maximum Lyapunov exponent: period-3 periodic window. (a) Limit point diagram, $b_{z}$ as a function of the driver amplitude $a$, for attractors $A_{1}$ and $A_{2}$, superimposed by the surrounding chaotic saddle (blue); (b) the same as (a), showing the conversion of the pre-crisis banded chaotic attractor (black) into the post-crisis banded chaotic saddle (red); (c) maximum Lyapunov exponent, $\lambda_{\max }$ as a function of $a$, for the attractor $A_{1}$. SNB denotes saddle-node bifurcation, and IC denotes interior crisis.

as periodic. A second attractor appears in a narrow range of control parameter indicated by $A_{2}$ in Fig. 5a. As seen in Fig. 5a, a saddle-node bifurcation (SNB) creates a pair of
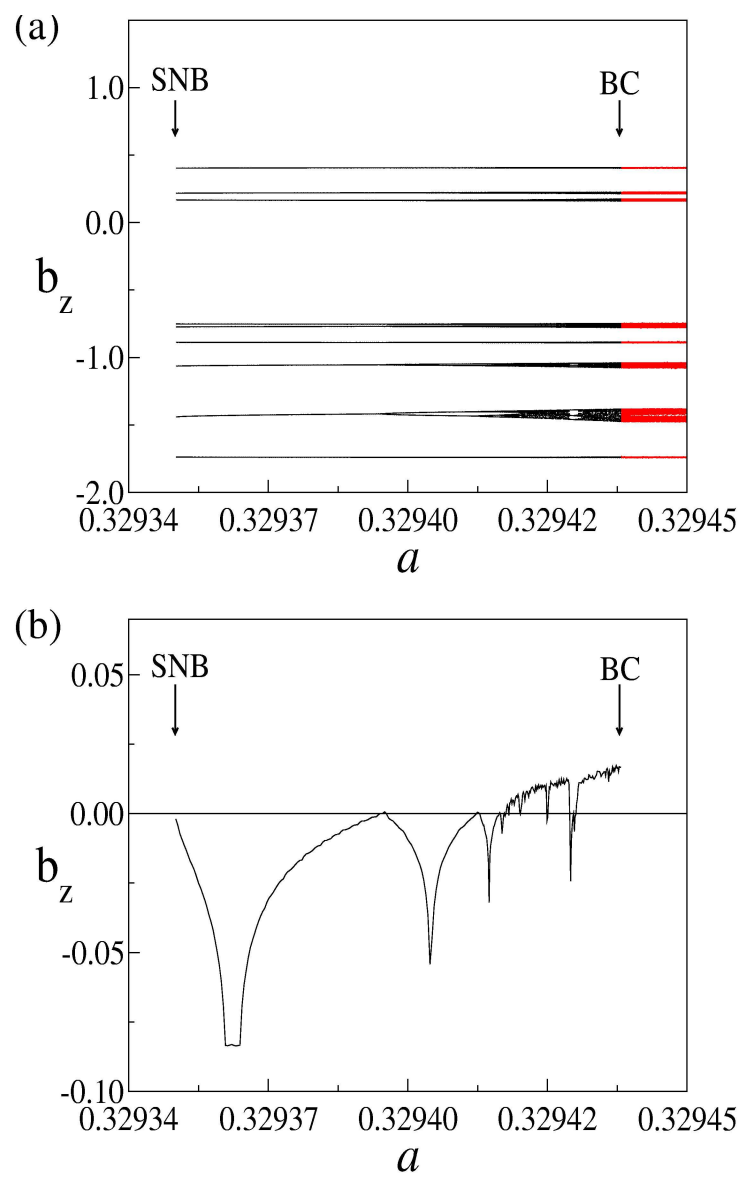

Fig. 3. Limit point diagram and maximum Lyapunov exponent: attractor $A_{2}$. (a) Limit point diagram, $b_{z}$ as a function of the driver amplitude $a$, for the attractor $A_{2}$, superimposed by the chaotic saddle (red) converted from the chaotic attractor $A_{2}$, after the boundary crisis; (b) the variation of maximum Lyapunov exponent $\lambda_{\max }$ as a function of $a$ for the attractor $A_{2}$. SNB denotes saddle-node bifurcation, and $\mathrm{BC}$ denotes boundary crisis.

stable (black solid line) and unstable periodic orbits (green dashed line) of period-3. An enlargement of the red rectangular region of Fig. 5a is given in Fig. 5b, which shows that a saddle-node bifurcation (SNB) creates a pair of stable (blue solid line) and unstable periodic orbits (red dashed line) of period-9. Figure $5 \mathrm{~b}$ also shows the first (PD-1) and second (PD-2) period-doubling bifurcations and the unstable periodic orbits (black dashed line) resulting from the respective bifurcations. At the boundary crisis (BC) indicated in Fig. 5b, the p-9 mediating unstable periodic orbit collides with the chaotic attractor evolved from the attractor $A_{2}$, leading to the destruction of $A_{2}$. At the interior crisis (IC) indicated in Fig. 5a, the same p-9 mediating unstable periodic orbit collides with the banded chaotic attractor evolved from the attractor $A_{1}$, leading to the formation of a strong chaotic attractor. 


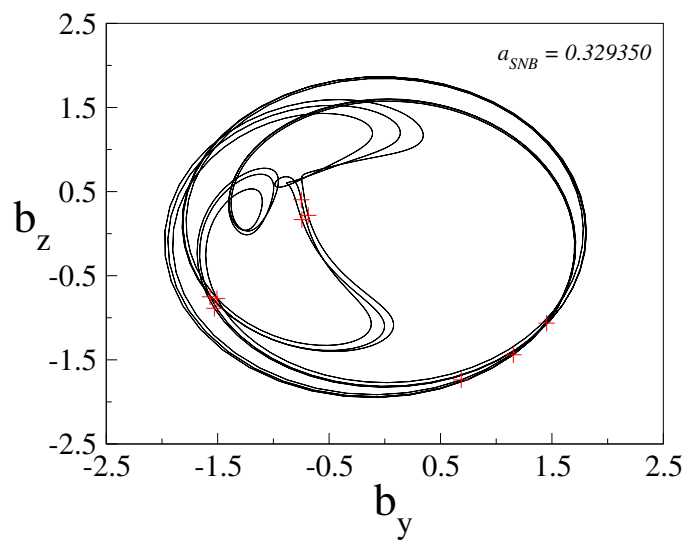

Fig. 4. Mediating unstable periodic orbit of period-9 created by a saddle-node bifurcation at $a_{S N B}=0.32935$. The solid line denotes the state-space trajectory, and the cross denotes the Poincare point.

\section{Saddle-node bifurcation}

As mentioned earlier, a local bifurcation known as saddlenode bifurcation takes place at $a_{\mathrm{SNB}}=0.321383$ in Fig. 2a, where a pair of period-3 stable and unstable periodic orbits are created. As the Alfvén system undergoes a transition from order to chaos via a saddle-node bifurcation, the surrounding chaotic saddle is converted into a chaotic attractor, as shown in Fig. 2a. Figure 6a shows the surrounding chaotic saddle (SCS) in the Poincaré plane for $a_{\mathrm{SNB}}=0.321383$, right after the saddle-node bifurcation. Figure $6 \mathrm{~b}$ shows the chaotic attractor (CA) for $a=0.321382 \lesssim a_{\mathrm{SNB}}$, just before the saddle-node bifurcation. The surrounding chaotic saddle (SCS) embedded in the chaotic attractor of Fig. $6 \mathrm{~b}$ is shown in Fig. 6c. Note from Figs. 6a and $\mathrm{c}$ that there are gaps in the surrounding chaotic saddles. The conversion from a chaotic saddle to a chaotic attractor, to the left of $a_{\mathrm{SNB}}$, is due to the creation of new unstable periodic orbits in the gap regions via the phenomenon of explosion (Robert et al., 2000; Szabó et al., 2000).

\section{Boundary crisis}

A global bifurcation known as boundary crisis (BC) takes place at $a_{\mathrm{BC}}=0.329437$ in Fig. 3a. Figure 7a shows the 9band chaotic attractor $A_{2}$ (CA, black) and the surrounding chaotic saddle (SCS, blue) in the Poincaré plane. The occurrence of boundary crisis is due to the collision of the chaotic attractor with the p-9 mediating unstable periodic orbit (M) created via a saddle-node bifurcation at $a_{\mathrm{SNB}}=0.321383$. Figure $7 \mathrm{~b}$ shows an enlargement of the rectangular region of Fig. 7a, indicating the collision of the chaotic attractor $A_{2}$ with the mediating unstable periodic orbit, its stable manifold (SM, thin line), and the surrounding chaotic saddle (SCS, (a)

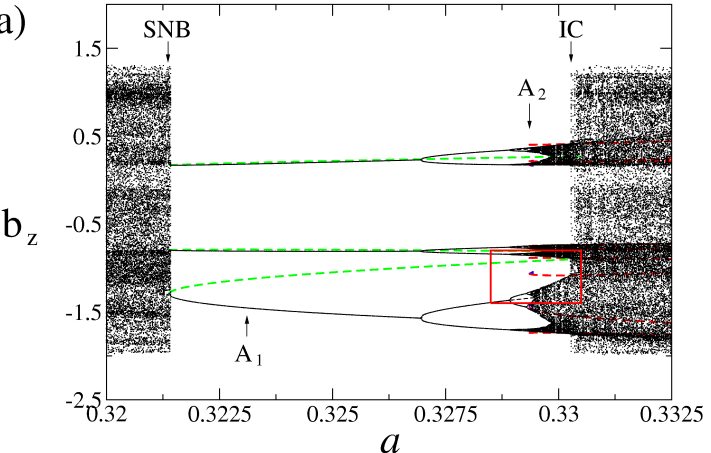

(b)

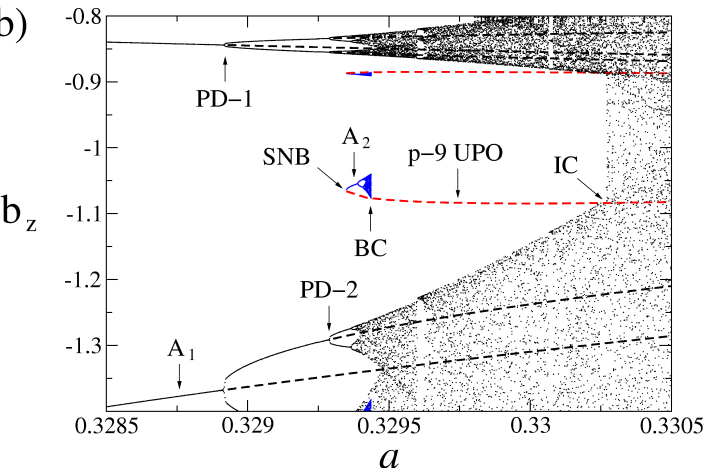

Fig. 5. Detailed bifurcation diagram of the period-3 periodic window. (a) Bifurcation diagram, $b_{z}$ as a function of the driver amplitude $a$, for attractors $A_{1}$ (balck) and $A_{2}$ (blue), p-3 unstable periodic orbit (green dashed line) and p-9 unstable periodic orbit (red dashed line) created by saddle-node bifurcations (SNB); (b) an enlargement of the red rectangular region indicated in panel (a). BC denotes boundary crisis and IC denotes interior crisis; PD-1/PD-2 denotes first/second period-doubling bifurcation of attractor $A_{1}$, respectively.

blue). Note that before the boundary crisis, two attractors $A_{1}$ and $A_{2}$ co-exist (see Fig. 2a), each with its own basin of attraction (Borotto et al., 2004); the stable manifold of the mediating unstable periodic orbit in Fig. $7 \mathrm{~b}$ forms the boundary between the two basins of attraction.

As the consequence of the chaotic attractor-chaotic saddle collision, after the onset of boundary crisis the chaotic attractor $A_{2}$ and its basin of attraction are destroyed, and the system converges to the attractor $A_{1}$, as seen in Fig. 2a. In Fig. 8a we plot the surrounding (SCS, blue) and banded (BCS, red) chaotic saddles after the boundary crisis at $a=0.329438 \gtrsim a_{\mathrm{BC}}$. After the boundary crisis, the pre-crisis 9-band chaotic attractor (CA) of Fig. 7a is converted into a 9-band chaotic saddle (BCS) of Fig. 8a. Figure 8b shows an enlargement of the rectangular region of Fig. 8a, indicating gaps in the banded chaotic saddle (BCS). 
(a)

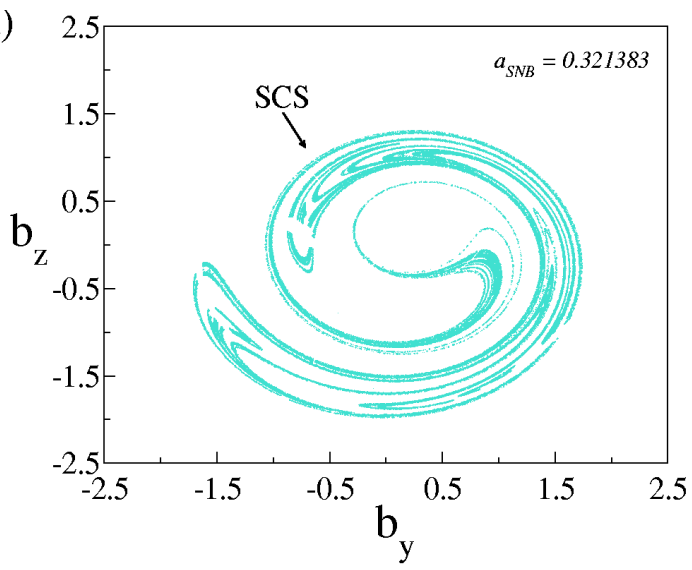

(b)

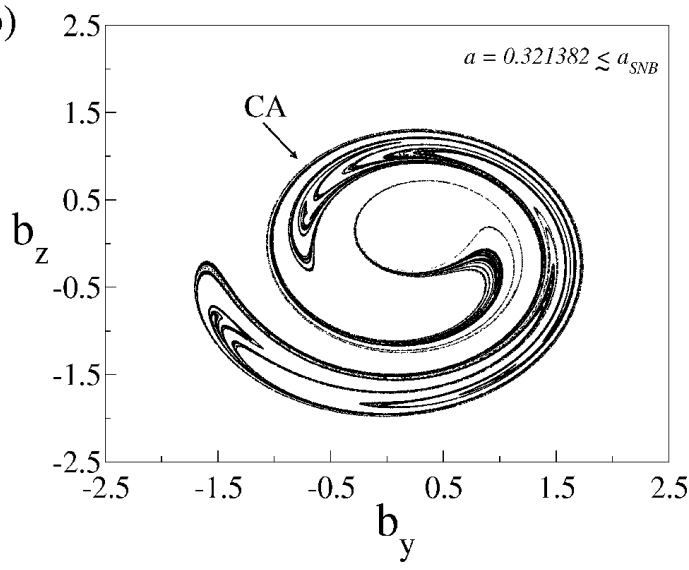

(c)

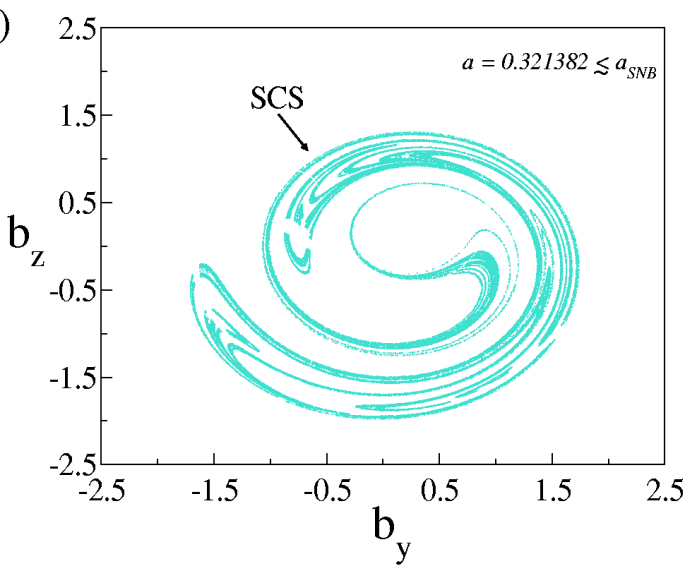

Fig. 6. Transition from a chaotic saddle to a chaotic attractor via a saddle-node bifurcation. (a) Post-SNB chaotic saddle at $a_{\mathrm{SNB}}=0.321383$; (b) pre-SNB chaotic attractor at $a=0.321382 \lesssim a_{\mathrm{SNB}}$; (c) pre-SNB chaotic saddle embedded in the chaotic attractor at $a=0.321382 \lesssim a_{\mathrm{SNB}}$.

\section{Interior crisis}

A global bifurcation known as interior crisis (IC) takes place at $a_{\mathrm{IC}}=0.330248$ in Fig. 2a. Figure 9a shows the weak chaotic attractor (WCA, black) and the surrounding chaotic (a)

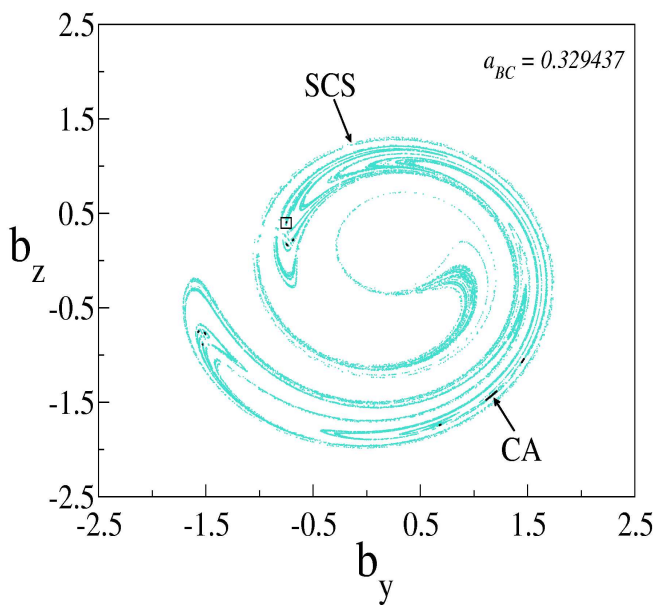

(b)

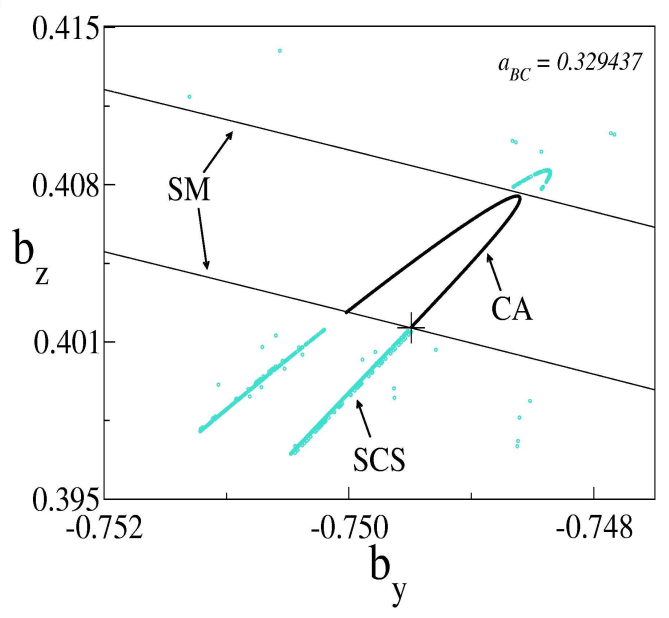

Fig. 7. Chaotic attractor-chaotic saddle collision at boundary crisis (BC) for $a_{\mathrm{BC}}=0.329437$. (a) Pre-BC surrounding chaotic saddle SCS (blue) and banded chaotic attractor CA (black); (b) a zoom of (a). The cross denotes a fixed point of the $p-9$ mediating unstable periodic orbit (M) and the thin line denotes its stable manifold (SM).

saddle (SCS) for $a_{\mathrm{IC}}$ in the Poincare plane. The occurrence of interior crisis is due to the collision of the weak chaotic attractor (WCA) with the p-9 mediating unstable periodic orbit $(\mathrm{M})$ created via a saddle-node bifurcation at $a_{\mathrm{SNB}}=0.321383$. Figures $9 \mathrm{~b}-\mathrm{c}$ are enlargements of the rectangular region in Fig. 9a.

Figure $9 \mathrm{~b}$ shows the Poincare points (cross) and the stable manifold (SM, light line) of the mediating unstable periodic orbit, the weak chaotic attractor (WCA), and the surrounding chaotic saddle (SCS, blue). It follows from Fig. 9b that the stable manifold of the mediating unstable periodic orbit forms the boundary between the banded and surrounding regions. Figure $9 \mathrm{c}$ shows the stable manifold (green) of the surrounding chaotic saddle and the weak chaotic attractor (WCA). Figures $9 \mathrm{~b}$ and $\mathrm{c}$ reveal that at the onset of crisis the chaotic attractor (WCA) collides head-on with the mediating 
(a)

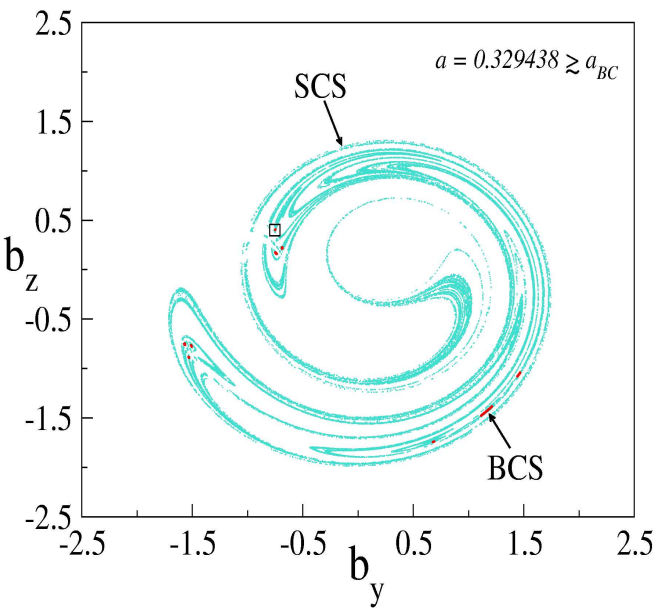

(b)

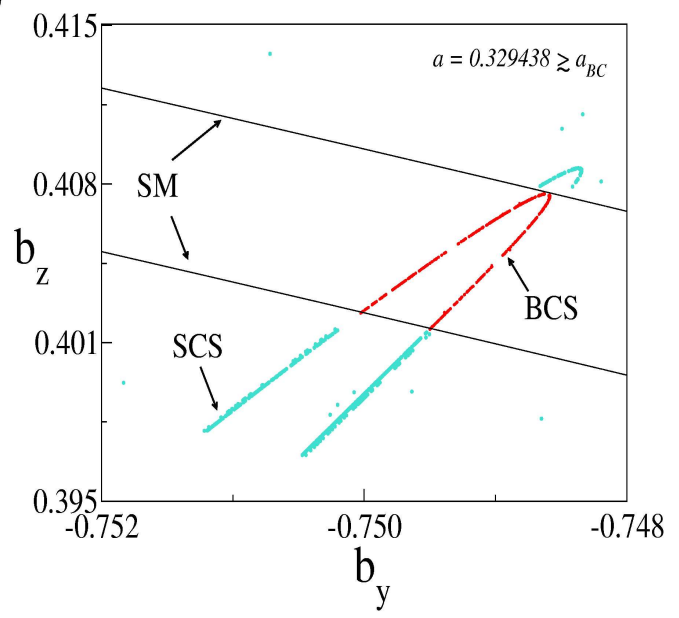

Fig. 8. Transition from a chaotic attractor to a chaotic saddle via a boundary crisis (BC). (a) Post-BC surrounding chaotic saddle (SCS, blue) and banded chaotic saddle (BCS, red) at $a=0.329438 \gtrsim a_{\mathrm{BC}}$; (b) a zoom of (a), the thin line denotes the stable manifold (SM) of the mediating unstable periodic orbit.

unstable periodic orbit and its associated stable manifold, as well as the surrounding chaotic saddle and its associated stable manifold.

As the result of the chaotic attractor-chaotic saddle collision, after the onset of interior crisis the pre-crisis weak chaotic attractor (WCA) turns into a post-crisis strong chaotic attractor (SCA), as shown by Fig. 10a for $a=0.330249 \gtrsim a_{\mathrm{IC}}$. Two chaotic saddles (surrounding and banded) are embedded in the strong chaotic attractor. In addition, the strong chaotic attractor contains a set of coupling unstable periodic orbits created by explosions after the onset of crisis. Figure $10 \mathrm{~b}$ shows the numerically found surrounding chaotic saddle (SCS, blue) and banded chaotic saddle (BCS, red). An enlargement of the rectangular region of Fig. 10b is plotted in Fig. 10c, where we also plot the stable manifold (SM, thin line) of the p-9 mediating unstable (a)

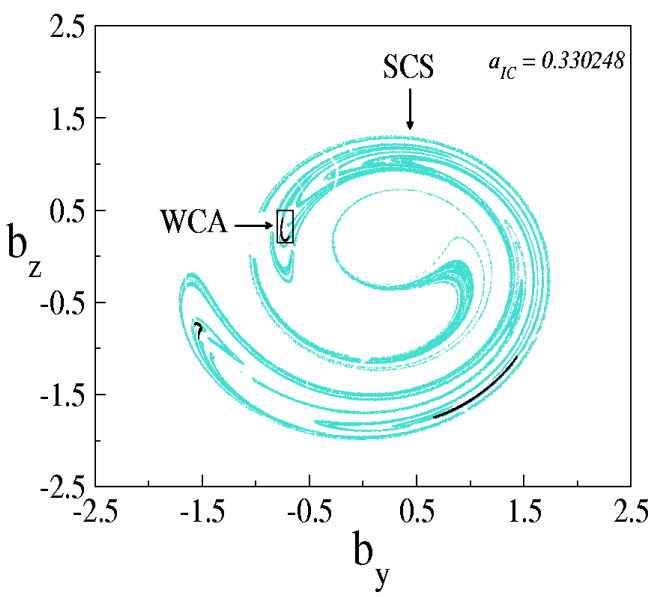

(b)

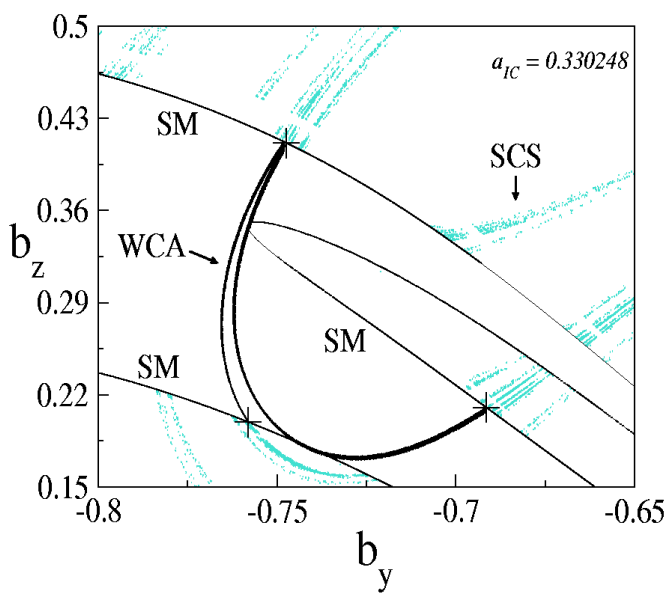

(c)

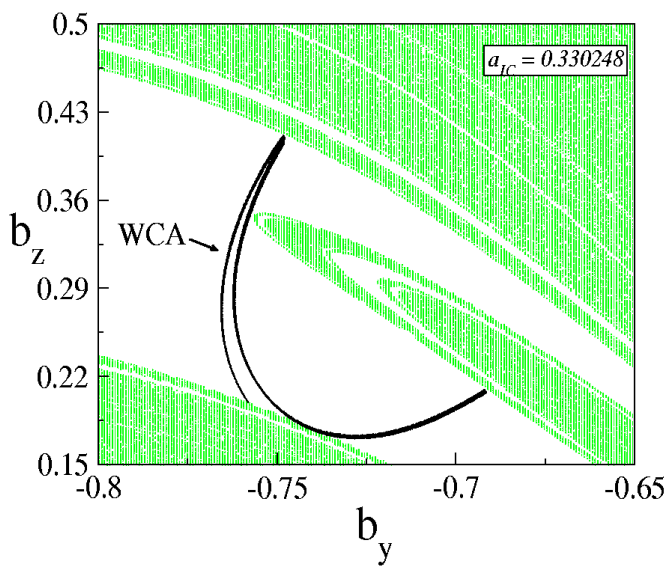

Fig. 9. Chaotic attractor-chaotic saddle collision at the Alfvén interior crisis for $a_{\mathrm{IC}}=0.330248$. (a) Pre-IC surrounding chaotic saddle (SCS) and weak chaotic attractor (WCA, black); (b) a zoom of (a) showing the collision of the weak chaotic attractor (WCA) with the mediating unstable periodic orbit (cross), its stable manifold (SM) and the surrounding chaotic saddle (SCS); (c) same as (b) showing the collision of the weak chaotic attractor (WCA) with the stable manifold (green) of the surrounding chaotic saddle. 
periodic orbit, which divides the surrounding and banded regions.

Evidently, Figs. 10b and c show that there are gaps inside the surrounding and banded chaotic saddles. These gaps are densely filled by uncountably many coupling unstable periodic orbits (C), created by explosions after the onset of interior crisis (Robert et al., 2000; Szabó et al., 2000), which have components in both surrounding and banded regions and are responsible for coupling the two regions. We find numerically one example of the coupling unstable periodic orbit. Figure 11a shows a coupling unstable periodic orbit of period-23 at $a=0.331>a_{\mathrm{IC}}$, which is created via an explosion at this value of $a$. Note that right after a crisis, the coupling unstable periodic orbits created by explosion in the gap regions have very long period with the period approaching infinity as $a$ approaches $a_{\mathrm{IC}}$ (Szabó et al., 2000). Since the control parameter in Fig. 11 is close to $a_{\mathrm{IC}}$, we were able to numerically find a coupling unstable periodic orbit with a long period-23.

An enlargement of the rectangular region of Fig. 11a is given in Fig. 11b. Figure 11 shows that the fixed points of the p-23 coupling unstable periodic orbit are located in the gap regions of both surrounding and banded chaotic saddles.

\section{Discussion and conclusion}

In this paper, we demonstrated that dynamical systems such as space plasmas are composed by unstable structures, namely, unstable periodic orbits and chaotic saddles. Within a periodic window, the chaotic saddle is responsible for the transient motion before the system converges to an attractor. In the chaotic regime, chaotic saddles are embedded in chaotic attractors. The transition from a chaotic saddle into a chaotic attractor occurs in both local bifurcation (e.g., saddlenode bifurcation) and global bifurcation (e.g., boundary crisis and interior crisis). For the saddle-node bifurcation and interior crisis, this transition is accompanied by explosions inside the gap regions of chaotic saddles which lead to the creation of new gap-filling unstable periodic orbits. Explosion only occurs for boundary crisis if the basin boundary is fractal, but do not occur for boundary crisis if the basin boundary is smooth (Robert et al., 2000). Due to the limitation of space, in this paper we only focused on the study of explosion and creation of gap-filling unstable periodic orbits for an interior crisis. In a future work, we will perform a detailed analysis of the system dynamics for a boundary crisis.

In this work, we applied both forward and backward time integrations to perform numerical analysis of unstable structures. In Figs. 7-11, chaotic attractors (CA, WCA, SCA) and chaotic saddles (SCS, BSC) are computed by forward time integrations, whereas the stable manifold (SM) of the mediating unstable periodic orbit is computed by backward time integrations based on the high-precision You-Kostelich-York (a)

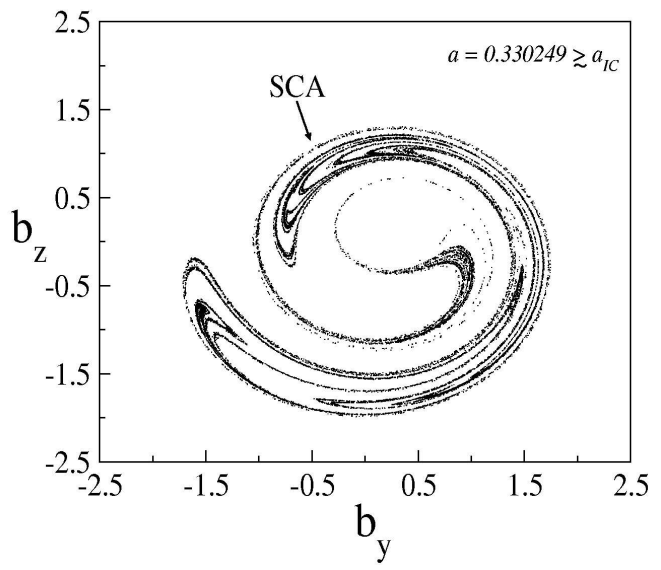

(b)

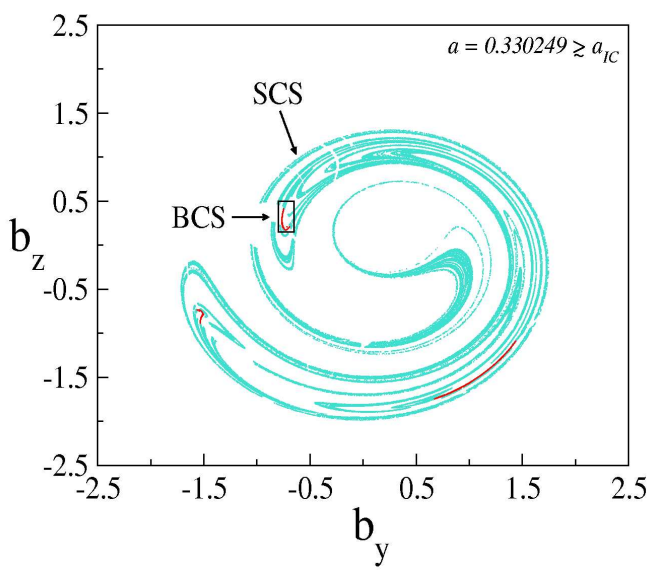

(c)

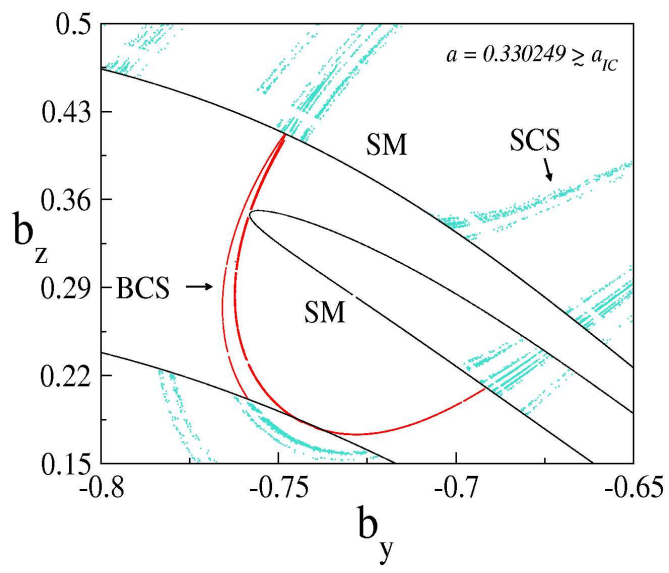

Fig. 10. Chaotic attractor and chaotic saddle after the Alfvén interior crisis at $a=0.330249 \gtrsim a_{\mathrm{IC}}$. (a) Poincaré map of the strong chaotic attractor (SCA); (b) the surrounding chaotic saddle (SCS, blue) and the banded chaotic saddle (BCS, red) embedded in the chaotic attractor of (a); (c) enlargement of the rectangular region of (b). SM (thin line) denotes the stable manifold of the mediating unstable periodic orbit of period-9. 
(a)

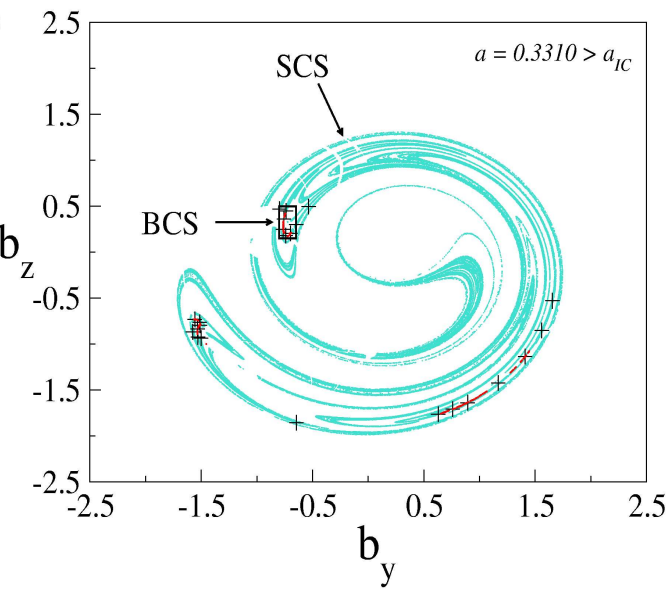

(b)

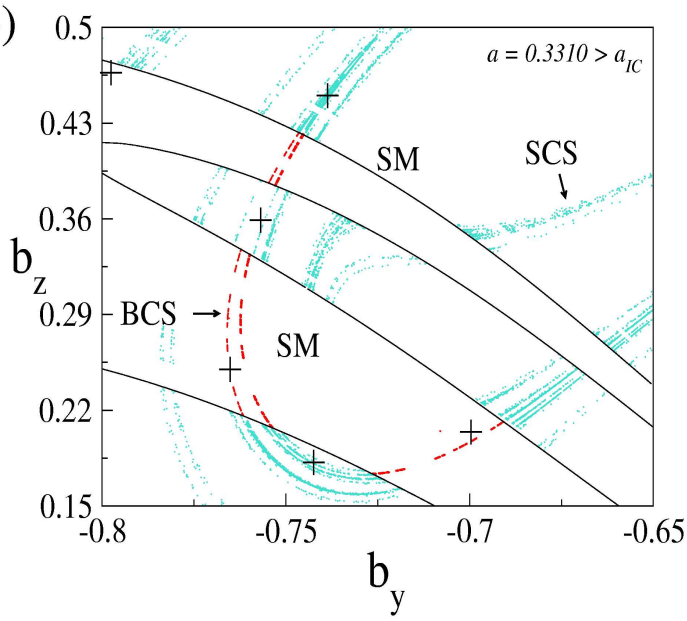

Fig. 11. Gap-filling unstable periodic orbit linking the banded and surrounding chaotic saddles after the Alfvén interior crisis at $a=0.3310$. (a) A coupling unstable periodic orbit of period-23 (cross) created via explosion at $a=0.3310>a_{\mathrm{IC}}$; (b) enlargement of the rectangular region of (a). The surrounding chaotic saddle (SCS) is indicated by blue, the banded chaotic saddle (BCS) is indicated by red, and SM (thin line) denotes the stable manifold of the mediating unstable periodic orbit of period- 9 .

algorithm (You et al., 1991), where the computed curve lies no further than a distance $\varepsilon$ from the true image curve. We set $\varepsilon=10^{-4}$. The You-Kostelich-York algorithm is useful for computing one-dimensional manifolds such as the present work. For two-dimensional stable and unstable manifolds, the efficient algorithm of Krauskopf and Osinga (1999) can be used.

The study of unstable structures in space plasmas can deepen our understanding of chaos in space plasmas such as the Alfvén intermittent turbulence in the solar wind (Chian et al., 1998; Bruno and Carbone, 2005). Nonlinear, intermittent Alfvén waves of solar and interplanetary origin can cause intense geomagnetic storms. The present work elu-
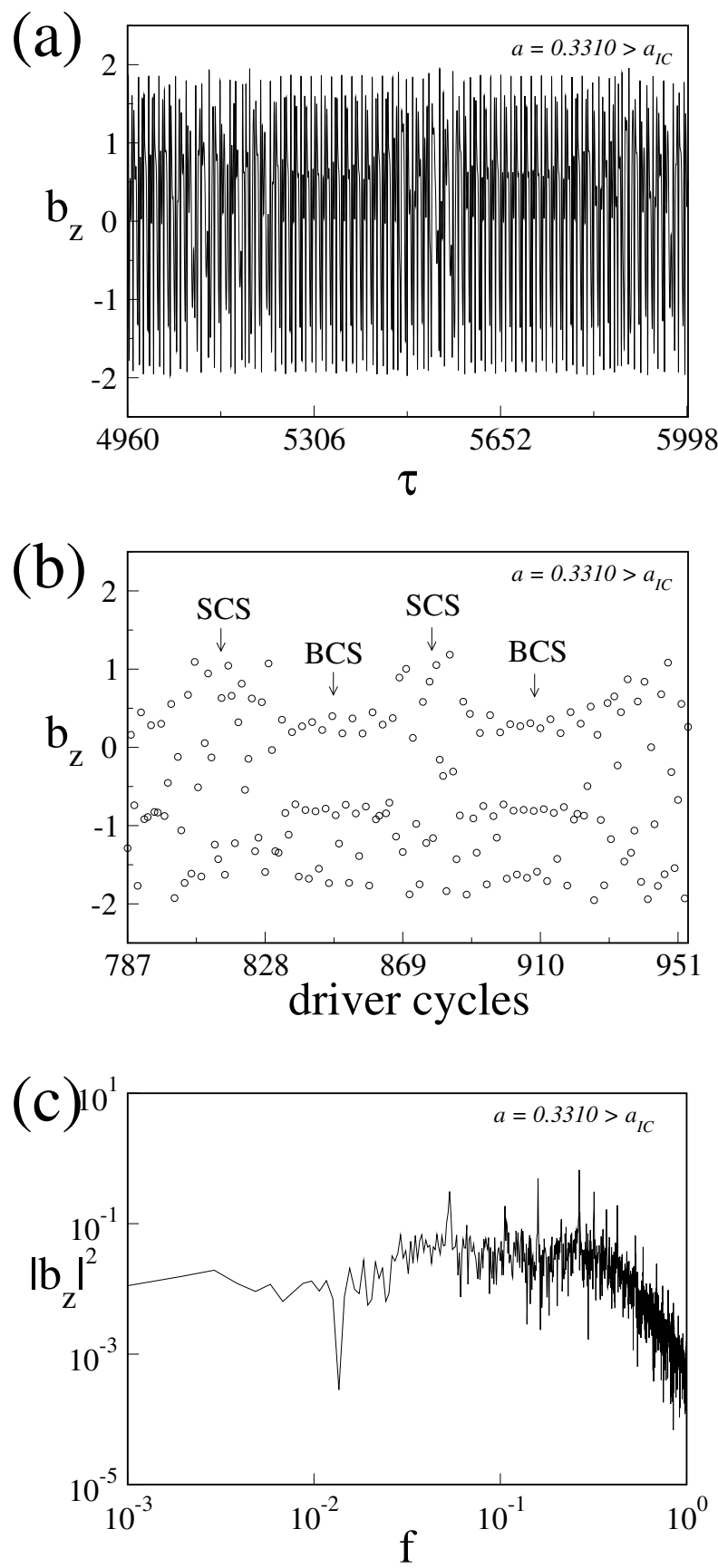

Fig. 12. Alfvén crisis-induced intermittency. (a) Time series $b_{z}$ as a function of $\tau$ for $a=0.3310$, (b) same time series as (a) plotted as a function of driver cycles, (c) power spectrum of (a). SCS denotes surrounding chaotic saddle and BCS denotes banded chaotic saddle.

cidates the fundamental dynamics and structures of Alfvén intermittency driven by chaos. Alfvén intermittency can be driven by a saddle-node bifurcation or by a global bifurcation such as interior crisis (Chian et al., 1998). For example, Figs. 12a and b show the time-series of Alfvén intermittency induced by the interior crisis studied in Sect. 6, 
whereby random regime switchings between laminar and bursty phases of the magnetic field fluctuations are observed. The corresponding power spectrum is given in Fig. 12c. This intermittent regime switching and power-law behavior of the power spectrum reproduce quite well the observation of Alfvén intermittent turbulence detected by the spacecraft in the solar wind (Bruno and Carbone, 2005). Figure 12b shows that the chaotic trajectory hops alternatively between the surrounding chaotic saddle (SCS) and the banded chaotic saddle (BCS) via the coupling unstable periodic orbits, as indicated by Figs. 10 and 11. In addition, it is evident from Figs. 12a and $b$ that during the laminar/bursty phases of the Alfvén intermittency, the chaotic trajectory is traversing the regions of the banded/surrounding chaotic saddle, respectively. The abrupt increase in the degree of chaoticity near the SNB and IC transitions in Fig. 2c implies that the interplanetary magnetic field fluctuations can change from periodic to aperiodic temporal patterns due to a saddle-node bifurcation, and change from low-amplitude to large-amplitude aperiodic temporal patterns due to an interior crisis.

The present work is based on the stationary solutions of the three-dimensional system Eqs. (2-4), which are a particular solution of the DNLS Eq. (1). This low-dimensional chaos approach enables us to perform a detailed analysis of the unstable structures of dynamical systems, which provides a valuable guide for studying high-dimensional chaos present in the spatiotemporal solutions of Eq. (1). Moreover, the methodology developed in this paper may serve as a guide for finding the unstable periodic orbit embedded in a welldeveloped fluid turbulence (Kawahara and Kida, 2001) and for understanding the role of chaotic saddles in the transition from laminar to turbulent flows in a pipe (Faisst and Eckhard, 2003).

In addition to space plasma applications, unstable structures are relevant to applications of chaos in the Earth's atmosphere and oceans such as the local predictability of the ElNiño Southern Oscillation (ENSO) (Samelson and Tziperman, 2001), the model of the thermolialine ocean circulation of the Atlantic (Titz et al., 2002) and barotropic waves (Kazantsev, 2001; Samelson, 2001a,b; Samelson and Wolfe, 2003; Selten and Branstator, 2004; Eccles et al., 2006). In conclusion, unstable periodic orbits and chaotic saddles are the key for monitoring and controlling the complex dynamics of the earth-ocean-space environment.

Acknowledgements. A. C.-L. Chian gratefully acknowledges the award of a visiting professor fellowship by Nagoya University and the kind hospitality of the Solar-Terrestrial Environment Laboratory. This work is supported by CNPq and FAPESP.

Edited by: J. Kurths

Reviewed by: W. M. Macek and another referee

\section{References}

Auerbach, D., Cvitanović, P., Eckmann, J.-P., Gunaratne, G., and Procaccia, I.: Exploring chaotic motion through periodic orbits, Phys. Rev. Lett., 58, 2387-2389, 1987.

Baumgartel, K.: Soliton approach to magnetic holes, J. Geophys. Res., 104, 28 295-28 308, 1999.

Borotto, F. A., Chian, A. C.-L., Hada, T., and Rempel, E. L.: Chaos in driven Alfvén systems: boundary and interior crises, Physica D, 194, 275-282, 2004.

Bruno, R. and Carbone, V.: The solar wind as a turbulence laboratory. Living Reviews in Solar Physics, http://solarphysics. livingreviews.org, 2005.

Buti, B.: Nonlinear Alfvén waves in inhomogeneous plasmas, Geophys. Res. Lett., 18, 809-812, 1991.

Chen, X. J. and Lam, W. K.: Inverse scattering transform for the derivative nonlinear Schrödiger equation with nonvanishing boundary conditions, Phys. Rev. E, 69, 066604, doi:10.1103, 2004.

Chian, A. C.-L., Borotto, F. A., and Gonzalez, W. D.: Alfvén intermittent turbulence driven by temporal chaos, Astrophys. J., 505, 993-998, 1998.

Chian, A. C.-L., Rempel, E. L., Macau, E. E., Rosa, E. E., and Christiansen, F.: High-dimensional interior crises in the Kuramoto-Sivashinsky equation, Phys. Rev. E, 65, 035203(R), doi:10.1103, 2002.

Chian, A. C.-L., Borotto, F. A., Rempel, E. L., Macau, E. E. N., Rosa, R. R., and Christiansen, F.: Dynamical systems approach to space environment turbulence, Space Sci. Rev., 107, 447-461, 2003.

Chian, A. C.-L., Rempel, E. L., and Rogers, C.: Complex economic dynamics: chaotic saddles, crisis and intermittency, Chaos, Solitons \& Fractals, 29, 1194-1218, 2006.

Christiansen, F., Cvitanović, P., and Putkaradze, V.: Spatiotemporal chaos in terms of unstable recurrent patterns, Nonlinearity, 10, 55-70, 1997.

Curry, J. H.: An algorithm for finding closed orbits, in: Global Theory of Dynamical Systems, edited by: Citecki, Z. and Robinson, C., p. 111, Springer-Verlag, Berlin, 1979.

Cvitanović, P.: Invariant measurement of strange sets in terms of cycles, Phys. Rev. Lett., 61, 2729-2732, 1988.

Dawson, S. P. and Fontan, C. F.: An analysis of unidimensional soliton gas models of magnetohydrodynamic turbulence in the solar wind, Astrophys. J., 348, 761-777, 1990.

Eccles, F. J. R., Read, P. L., and Haine, T. W. N.: Synchronization and chaos control in a periodically forced quasi-geostrophic two-layer model of baroclinic instability, Nonlin. Processes Geophys., 13, 23-39, 2006, http://www.nonlin-processes-geophys.net/13/23/2006/.

Faisst, H. and Eckhardt, B.: Traveling waves in pipe flow, Phys. Rev. Lett., 91, 224 502, doi:10.1103, 2003.

Ghosh, S. and Papadopoulos, K.: The onset of Alfvén turbulence, Phys. Fluids, 30, 1371-1387, 1987.

Grebogi, C., Ott, E., and Yorke, J. A.: Crises, sudden changes in chaotic attractors, and transient chaos, Physica D, 7, 181-200, 1983.

Green, K. and Krauskopf, B.: Global bifurcation and bistability at the locking boundaries of a semiconductor laser with phaseconjugate feedback, Phys. Rev. E, 66, 016220, doi:10.1103, 2002. 
Hada, T., Kennel, C. F., and Buti, B.: Stationary nonlinear Alfvén waves and solitons, J. Geophys. Res., 94, 65-77, 1989.

Hada, T., Kennel, C. F., Buti, B., and Mjølhus, E.: Chaos in driven Alfvén systems, Phys. Fluids B, 2, 2581-2590, 1990.

Harrison, P. K., Tattersall, J. E. H., and Clement R. A.: Periodic orbit analysis reveals subtle effects of atropine on epileptiform activity in the guinea-pig hippocampal slice, Neuroscience Lett., 357, 183-186, 2004.

Ishiyama, K. and Saiki, Y.: Unstable periodic orbits and chaotic economic growth, Chaos, Solitons \& Fractals, 26, 33-42, 2005.

Kantz, H.: A robust method to estimate the maximal Lyapunov exponent of a time-series, Phys. Lett. A, 185, 77-87, 1994.

Kato, S. and Yamada, M.: Unstable periodic solutions embedded in a shell turbulence, Phys. Rev. E., 68, 025302(R), doi:10.1103, 2003.

Kawahara, G. and Kida, S.: Periodic motion embedded in plane Couette turbulence: regeneration cycle, J. Fluid Mech., 449, 291-300, 2001.

Kawahara, G.: Laminarization of minimal plane Couette flow: going beyond the basin of attraction of turbulence, Phys. Fluids, 17, 041702, doi:10.1063, 2005.

Kazantsev, E.: Sensitivity of the attractor of the barotropic ocean model to external influences, Nonlin. Processes Geophys., 8, 281-300, 2001, http://www.nonlin-processes-geophys.net/8/281/2001/.

Kennel, C. F., Buti, B., Hada, T., and Pellat, R.: Nonlinear, dispersive, elliptically polarized Alfvén waves, Phys. Fluids, 31, 19491961, 1988.

Khabibrakhmanov, I. K., Galeev, A. A., and Galinsky, V. L.: Collisionless parallel shocks, J. Geophys. Res., 98, 1293-1301, 1993.

Krauskopf, B. and Osinga, H.: Two-dimensional global manifolds of vector fields, Chaos, 9, 768-774, 1999.

Krishan, V. and Nocera, L.: Relaxed states of Alfvénic turbulence, Phys. Lett. A, 315, 389-394, 2003.

Lashkin, V. M.: Generation of solitons by a boxlike pulse in the derivative nonlinear Schrödinger equation with nonvanishing boundary conditions, Phys. Rev. E, 71, 066613, doi:10.1103, 2005.

Laveder, D., Passot, T., and Sulem, P. L.: Transverse collapse of low-frequency Alfvén waves, Physica D, 152, 694-740, 2001.

Li, T. Y. and Yorke, J. A.: Period 3 implies chaos, American Math. Monthly, 82, 985-992, 1975.

Machida, S., Spangler, S. R., and Goertz, C. K.: Simulation of amplitude-modulated circularly polarized Alfvén waves for betaless-than one, J. Geophys. Res., 92, 7413-7422, 1987.

Medvedev, M. V. and Diamond, P. H.: Fluid models for kinetic effects on coherent nonlinear Alfvén waves: 1. fundamental theory, Phys. Plasmas, 3, 863-873, 1996.

Mjølhus, E.: On the modulational instability of hydromagnetic waves parallel to the magnetic field, J. Plasma Phys., 16, 321334, 1976.

Nusse, H. E. and Yorke, J. A.: A procedure for finding numerical trajectories on chaotic saddles, Physica D, 36, 137-156, 1989.

Ott, E.: Chaos in Dynamical Systems, Cambridge University Press, Cambridge, 1993.

Passot, T. and Sulem, P. L.: Filamentation instability of long Alfvén waves in warm collisionless plasmas, Phys. Plasmas, 10, 39143921, 2003.

Rempel, E. L. and Chian, A. C.-L.: Alfvén chaotic saddles, Int. J.
Bifurcation Chaos, 14, 4009-4017, 2004.

Rempel, E. L., Chian, A. C.-L., Macau, E. E., and Rosa, R. R.: Analysis of chaotic saddles in low-dimensional dynamical systems: the Kuramoto-Sivashinsky equation, CHAOS, 14, 545556, 2004a.

Rempel, E. L., Chian, A. C.-L., Macau, E. E., and Rosa, R. R.: Analysis of chaotic saddles in low-dimensional dynamical systems: the derivative nonlinear Schrodinger equation, Physica D, 199, 407-424, 2004b.

Rempel, E. L., Chian, A. C.-L., Preto, A. J., and Stephany, S.: Intermittent chaos driven by nonlinear Alfvén waves, Nonlin. Processes Geophys., 11, 691-700, 2004c.

Rempel, E. L. and Chian, A. C.-L.: Intermittency induced by attractor-merging crisis in Kuramoto-Sivashinsky equation, Phys. Rev. E, 71, 016203, doi:10.1103, 2005.

Robert, C., Alligood, K. T., Ott, E., and Yorke, J. A.: Explosions of chaotic sets, Physica D, 144, 44-61, 2000.

Ruderman, M. S.: DNLS equation for large-amplitude solitons propagating in an arbitrary direction in a high-beta Hall plasma, J. Plasma Phys., 67, 271-276, 2002.

Samelson, R. M.: Periodic orbits and disturbance growth for baroclinic waves, J. Atmos. Sci., 58, 436-450, 2001a.

Samelson, R. M.: Lyapunov, Floquet, and singular vectors for baroclinic waves, Nonlin. Processes Geophys., 8, 439-448, $2001 \mathrm{~b}$.

Samelson, R. M. and Tziperman, E.: Instability of the chaotic ENSO: the growth-phase predictability barrier, J. Atmos. Sci., 58, 3613-3625, 2001.

Samelson, R. M. and Wolfe, C. L.: A nonlinear baroclinic wavemean oscillation with multiple normal mode instabilities, J. Atmos. Sci., 60, 1186-1199, 2003.

Selten, F. M. and Branstator, G.: Preferred regime transition routes and evidence for an unstable periodic orbit in a baroclinic model, J. Atmos. Sci., 61, 2267-2282, 2004.

Spangler, S. R. and Sheerin, J. P.: Properties of Alfvén solitons in a finite-beta plasma, J. Plasma Phys., 27, 193-198, 1982.

Spangler, S. R. and Plapp, B. B.: Characteristics of obliquely propagating, nonlinear Alfvén waves, Phys. Fluids B, 4, 3356-3370, 1992.

Szabó, K. G. and Tél, T.: Thermodynamics of attractor enlargement, Phys. Rev. E, 50, 1070-1082, 1994a.

Szabó, K. G. and Tél, T.: Transient chaos as the backbone of dynamics of strange attractors beyond crisis, Phys. Lett. A, 196, 173-180, 1994b.

Szabó, K. G., Lai, Y.-C., Tél, T., and Grebogi, C.: Topological gap filling at crisis, Phys. Rev. E, 61, 5019-5032, 2000.

Titz, S., Kuhlbrodt, T., and Feudel, U.: Homoclinic bifurcation in an ocean circulation box model, Int. J. Bifurcation Chaos, 12, 869-875, 2002.

van Veen, L., Kida, S., and Kawahara, G.: Periodic motion representing isotropic turbulence, Fluid Dyn. Res., 38, 19-46, 2006.

Verheest, F. and Buti, B.: Parallel solitary Alfvén waves in warm multispecies beam-plasma systems. 1., J. Plasma Phys., 47, 1524, 1992.

Verheest, F., Cattaert, T., Dubinin, E., Sauer, K., and McKenzie, J. F.: Whistler oscillitons revisited: the role of charge neutrality?, Nonlin. Processes Geophys., 11, 447-452, 2004, http://www.nonlin-processes-geophys.net/11/447/2004/.

Wolf, A., Swift, J. B., Swinney, H. L., and Vastano, J. A.: Determining Lyapunov exponents from a time series, Physica D, 16, 
285-317, 1985.

Wu, C. C.: MKDVB and CKB shock waves, Space Sci. Rev., 107, 403-421, 2003.

You, Z., Kostelich, E. J., and Yorke, J. A.: Calculating stable and unstable manifolds, Int. J. Bifurcation Chaos, 1, 605-623, 1991.
Zoldi, S. M. and Greenside, H. S.: Spatially localized unstable periodic orbits of a high-dimensional chaotic system, Phys. Rev. E, 57, 2511-2514(R), 1998. 